\section{THE DIAGNOSIS OF GALL-STONES. ${ }^{1}$}

BY JAMES SHERREN, F.R.C.S. ENG., SURGEON TO THE LONDON HOSPITAL, ETC.

THE recognition of the cause of pain in the right upper quadrant of the abdomen is a problem that continually confronts us and often baffles our diagnostic powers. Until recent years it was usual to look upon violent attacks of colic followed by jaundice as necessary to the diagnosis of gallstones and as indicative of their presence. Judging from the late period at which many cases are sent for operation this view is still prevalent.

No one speaking on the subject of gall-stones can omit to pay tribute to the work done by Mayo Robson and by Moynihan in attempting to dispel this common error, and especially to the latter in pointing out the significance of the "prodromal " symptoms of gall-stones. ${ }^{2}$

Colic is a late symptom of gall-stones, and even when followed by jaundice does not necessarily indicate their presence. I wish to direct your attention to the diagnosis between the colic due to gall-stones and that due to moveable kidney and to the early symptoms produced by gall-stones, referred to the stomach and called "dyspepsia."

Although it is now many years since the occurrence of jaundice in association with moveable kidney was recognised, the impossibility in many cases of its differential diagnosis apart from operation is a subject which has not received the attention that it deserves. Surgeons generally seem to have overlooked or paid little attention to the subject. Mayo Robson in his book, ${ }^{3}$ in the section on diagnosis, makes no mention of it, but in the section on kinking of the bile-ducts (p. 139) he states that "those cases of catarrh of the gallbladder producing attacks resembling cholelithiasis without gall-stones but associated with a moveable right kidney, probably owe their origin to kinking of the bile-ducts due to dragging by the displaced kidney." Two cases are given in the appendix. Moynihan, in the chapter on differential diagnosis in his book, ${ }^{ \pm}$under a list of the various disease which nay be confounded with gall-stones, writes: "Diseases of the right kidney, more especially calculus, or that intermittent kinking of the ureter or the vessels of the kidney which cause Dietl's crises." Later he writes : " "Renal colio or undue renal mobility upon the right side has in some instances caused a confusion in diagnosis." Neither under the heading of diagnosis nor in the chapter on jaundice in either work is an account given of the cases in which the symptoms are indistinguishable from gall-stones or of those with obstructive jaundice. Bland-Sutton ${ }^{6}$ mentions the simulation of the colic associated with gall-stones by "sudden distension of the renal pelvis with urine (acute hydronephrosis) when the kidney is sufficiently mobile to allow the ureter to become kinked"; and again : "A mobile kidney will cause jaundice by dragging on the duodenum." In the most modern text-book of surgery in the English language ${ }^{8}$ in the article written by W. J. and C. H. Mayo, no mention is made of it. The only satisfactory account that I have been able to find is in Rolleston's exhaustive monograph. ${ }^{3} \mathrm{He}$ writes: "A floating kidney may not only produce symptoms like hepatic colic and induce jaundice, but the displaced kidney may readily be mistaken for a distended gall-bladder."

The association of jaundice with moveable kidney appears to have been first recorded by Brochin in $1854,{ }^{10}$ next by Litten ${ }^{11}$ in 1880 . Landau, ${ }^{12}$ in his classical monograph, stated that he had observed it in three cases and wrote as follows: "It is, however, certain that women who have møveable kidneys are more prone than others to jaundice, but only because they more frequently suffer from gastroduodenal catarrh ...... and because a moveable kidney not

1 A paper read before the Hunterian Society on March 8th, 1911.

2 Moynihan: Brit. Med. Jour., Nov. 28th, 1898. p. 230 . Gall-stones and their Surgical Treatment,

Gall-stones and Diseases of the Bile-ducts, second edition, 1910, p. 183. 7 Ibid., p. 180

8 Keen's Surcery, 1908 vol iii.

9 Diseases of the Liver, Gall-bladder, and Bile-ducts, 1905, pp. 546, 730. 10 Gazette des Hôpitaux.

11 Quoted by Landau, Moveable Kidney in Women, New Sydenham Society's Transactions, vol. cx., p. 288. infrequently contracts adhesions to the gall-bladder." After this date isolated cases were recorded by Hale White, ${ }^{13}$ Clennell Fenwick, ${ }^{1 t}$ Maclagan and Treves, ${ }^{15}$ Macpherson Lawrie. ${ }^{16}$ In 1902 two important papers appeared which have been generally overlooked. J. Hutchinson, jun., ${ }^{17}$ described two cases in which obstructive jaundice due to moveable kidney was cured by fixation of the kidney, and demonstrated the cause of the symptoms. Marwedel ${ }^{13}$ reviewed 14 published cases and reported four cases of his own bearing on the question, and in his summing-up states "A moveable right kidney may produce all the symptoms of cholelithiasis - colic with or without jaundice in the absence of diseases of the biliary passages." In $1908^{19} \mathrm{I}$ published a short history of two cases drawing attention to the difficulties in diagnosis, and in 1909 Sir Watson Cheyne ${ }^{20}$ mentions it in a paper on Moveable Kidney. Cases of this nature appear to have fallen to the lot of the general surgeon particularly, for Sir Henry Morris" wrote: "Transient attacks of jaundice are said not to be infrequent, but I have never personally witnessed them."

Not only may the symptoms produced by moveable kidney simulate those due to gall-stones, but the two conditions not infrequently co-exist. I should like to illustrate some points by quoting cases that have been under my care.

The first was a woman, aged 22 years, whom I saw in May, 1903, with obstructive jaundice of a week's duration. She was married and had had three children. For eight months she had noticed a moveable lump in her right side, and had had a dragging pain on exertion. During the four months before coming under my care she had had three attacks of "biliary colic" ; jaundice followed the last of these. Marked obstructive jaundice was present and the gall-bladder formed a definite tumour reaching to the mid-point between the umbilicus and ninth costal cartilage and the right kidney was moveable. I explored and fixed the right kidney. The jaundice gradually disappeared and the gall-bladder could not be felt 14 days after operation.

I am in the habit of teaching that obstructive jaundice with a distended gall-bladder in young women is usually due to mobile right kidney. The next case I saw in November, 1905.

A young woman, 26 years of age, was sent to me with a history of obstructive jaundice of five weeks' duration. For 18 months she had been having sudden attacks of pain in the right hypochondrium, accompanied by vomiting. She was markedly jaundiced. The right kidney was very mobile, but the gall-bladder was not palpable. I operated and found a shrunken gall-bladder with dilated common and cystic ducts. I removed 40 stones from the ducts, not one larger than pea, and drained the common bile-duct. She made an un. interrupted recovery. As she complained of pain due to her mobile kidney I fixed this later, and she is now in perfect health.

I have quoted these two histories together as illustrating, first, a case in which before operation it could reasonably be supposed that the kidney alone was responsible for the obstructive jaundice. In the second case the gall-bladder was not distended and the diagnosis of stones in the common duct was made. It is probable that the moveable kidney was here a predisposing cause of gall -stones.

It is, however, unusual to find jaundice present. Less severe cases are more common. The following is an example of the usual type.

In December, 1908, I saw a woman, aged 46 years, with the following history. During the past five months she had had three severe attacks of pain starting in the right hypo. chondrium and passing round to the right, accompanied by vomiting. The first attack was followed by jaundice. found a very mobile right kidney. The gall-bladder was not enlarged and there had been no urinary symptoms. I thought that the mobile kidney would account for all the symptoms, but was by no means certain. I followed out my usual plan and made a small incision through the right rectus muscle and explored the gall-bladder and biliary

13 Brit. Med. Jour., 1892, vol. i., p. 233.

14 The Lancex, Nov. 11th, 1899, p. 1296

15 THE LANCET, Jan, 6th, 1900, p. 15.

17 Brit. Med. Jour, 1901, vol. i., p. 15.

1s Beiträge zur Ílinischen Chirurgie, Band xxxiv., 1902, S. 478

19 Clinical Journal, Oct. 14th, 1908, p. 12 .

20 THE LANCET, April 24th, 1909 , p. 1155 .

21 Surgical Diseases of the Kidney and Ureter, vol. i., p. 113 
passages. The gall-bladder was full of bile. As I was unable to empty it I opened it; no stones were present and its mueous membrane was healthy. I closed the gallbladder and fixed the kidney and she has remained well.

It is a rule that should never be neglected in gall-bladder surgery that if the gall-bladder cannot be emptied so that epen small stones could be palpated it must be opened and the contents examined. In this way I found calculi in two cases associated with moveable kidney that I should otherwise have overlooked. Among 13 cases in which symptoms suggestive of cholelithiasis were associated with a moveable kidney, all except one were women. Gall-stones were present in two only. In none had urinary symptoms occurred. In addition to these 13 cases I fixed the moveable kidney of a patient whose gall-bladder had been explored on the diagnosis of gall-stones two years previously. This was 20 months ago, and the patient has remained well since. I have also fixed the kidney of a patient in whom a biliary fistula existed as the result of draining the gall-bladder for "gall-bladder symptoms." The fistula closed after the kidney had been fixed. With regard to the differential diagnosis, this is difficult, and, I believe, in most cases impossible. Even when the symptoms are due to moveable kidney alone, tenderness will be present in the gall-bladder region during the attack, and Murphy's sign may be marked; tenderness may also be present over the lower ribs posteriorly. When jaundice exists, the fact that the gall-bladder is distended should make us suspect that the moveable kidney is the cause. It most always be remembered that the two conditions may coexist. Rolleston ${ }^{22}$ states that "the diagnosis depends on the detection of a floating kidney, for the symptoms, biliary colic and jaundice, are the same as those of cholelithiasis. If the symptoms persist after the floating kidney has been efficiently and successfully treated, it is probable that there is cholelithiasis in addition." There is no means, apart from operation, by which the diagnosis can be made. If the attacks are due to moveable kidney they rarely occur at night, while attacks of colic due to gall-stones frequently wake the patient in the early hours of the morning.

In operating upon cases of this nature in which the diagnosis is doubtful, a vertical incision should be made through the fibres of the right rectus muscle, the biliary passages examined, and the gall-bladder opened if necessary. If no stones are present the kidney should be fixed through a posterior incision. If stones are present, I believe that the kidney should also be fixed at the same time if the condition of the patient will admit of it. The results of treatment have been most satisfactory.

Difficulty is often experienced in the diagnosis of diseases of the stomach and duodenum from gall-stones. The symptoms produced by the presence of stones in the gallbladder are called "indigestion," the underlying cause of which is often considered to be "gastritis," gastric or duodenal ulcer. It is not zet realised that indigestion is the most common first symptom of gall-stones. In looking into most of the text-books dealing with diseases of the stomach or duodenum or with gall-stones, I have been struck by the fact that the greatest stress is laid on the diagnosis when biliary colic occurs. But this is a late symptom. It is in the absence of these attacks and in the period before they occur that diagnosis is so urgently necessary for safe and efficient treatment, and in some cases so difficult.

Of English writers, Moynihan ${ }^{23}$ alone has laid stress on these prodromal symptoms, and draws a distinction between biliary colic and pain apart from this and discusses the early symptoms. He says ${ }^{24}$ : "The chief difficulty will be in distinguishing cases of duodenal or gastric ulcer from cholelithiasis. In some cases it is impossible." With this I am in absolute agreement.

Rolleston, ${ }^{25}$ in discussing differential diagnosis, does not mention these cases. He states that the "pain of gastric ulcer has a direct relationship to food," so has the pain in certain cases of cholelithiasis. Again, "the pain of gall stone colic is more to the right of the abdomen than that of gastric ulcer, and may be absent for long periods. That of ulcer constantly recurs after food." The pain in chole lithiasis may be after every meal, and in cases of chronic

22 Diseases of the Liver, Gall-bladder, and Bile-ducts, 1905. ${ }^{23}$ Gall-stones and their Surgical Treatment, second edition, 1905 p. 149 et seq.

24 Ibid., p. 176 et seq.
25 Diseases of the Liver, Gall-bladder, and Bile-ducts, 1905, p. 730. ulcer of both stomach and duodenum there are usually wellmarked periods in which the patient is nearly or quite well. In another place he states ${ }^{26}$ : "Indigestion is a frequent result of gall-stones, so-called irregular biliary colic not infrequently manifests itself as dyspepsia and may be due to adhesions between gall-bladder and stomach ...... continued indigestion should be regarded as an important factor in the production of gall-stones." Deaver and Ashurst ${ }^{27}$ state : "Gall-stone colic and cholecystitis are usually sufliciently distinguished by their symptoms and physical signs, as well as by the extreme irregularity of the attacks of gall-stone colic, which bear no relation whatever to the ingestion of food, and which recur with no persistence after each meal, as does the pain due to gastric ulcer. Moreover, in the intervals of biliary colic the digestion is apparently in perfect order, and pain is absent."

It is quite evident that the importance of the digestive symptoms of gall-stones and the difficulties in diagnosis from diseases of the stomach has not been fully realised.

For the purpose of this paper I have gone through the notes of all the cases of gall-stones that I have operated on in the last two years-43 in number. Of these, 26 had had indigestion, for which they had been under medical care for long periods ; one patient after being treated as gastric ulcer was sent into hospital on the occurrence of her first attack of colic as a case of perforated gastric ulcer. Another patient had had gastro-enterostomy performed without relief; at operation I removed 140 small stones from the gall-bladder and one from the common bile-duct. Of these 26 cases, 20 had had attacks of biliary colic after periods of indigestion ranging from 5 months to 40 years; in the majority of the cases the history of indigestion was of over 5 years' duration.

The "dyspepsia" falls into two groups: (1) those in which the symptoms suggest the prodromal symptoms of gall. stones ; and (2) those rare cases in which a certain diagnosis from duodenal ulcer is impossible. Taking the groups in order, Group 1 is the more important, for $I$ believe it is at this stage that the operation should be performed before biliary colic has occurred. Operation is free from risk while the stones are still in the gall-bladder and no complications have ensued. Out of the 20 patients upon whom I operated after attacks of colic following a long period of dyspepsia, in no less than nine had I to remove calculi from the common bile duct, and in three of these I removed stones from the hepatic ducts as well. The following is a typical case of dyspepsia due to gall-stones.

A man, aged 46 years, was sent to me in September, 1909, with a history of indigestion extending over 20 years. During the first 18 years this came on in attacks of from two to three weeks' duration with from two to three weeks' interval. The pain came on from half an hour to two hours after a meal, lasted four or five hours, was situated in the epigastric region, and was accompanic $d$ by a feeling of distension "as if he would burst." It was not associated with vomiting and was never relieved by taking food but often relieved by " belching." It often woke him at 3 A.M. There was never any colic. The condition had been treated as "gastritis" by lavage and as gastric ulcer. I diagnosed gall-stones on account of the feeling of distension accompanying the pain, and because of the time at which the pain woke him and the fact that the pain was never relieved by taking food. At operation I found the gall-bladder free from adhesions and containing hundreds of small calculi. The patient lost his gastric symptoms and has remained well.

Another example was the case of a man, aged 24 years, upon whom I operated in August, 1908, and who was sent to me as suffering from duodenal ulcer. For five years he had been having epigastric pain with a feeling of distension two hours after his meals. Until 18 months previously this was in attacks of about a month's duration at irregular intervals but had been present every day since. The pain was never associated with vomiting and never relieved by taking food. Ten days before I saw him jaundice was noticed; it came on painlessly and he had never had an attack of colic. On examination no tenderness was manifest except over the gall-bladder region; the gall-bladder was not felt. I diagnosed gall-stones and not chronic duodenal ulcer with obstructive jaundice. Had it been the latter, pain would

26 Ibid., p. 709

27 Surgery of the Upper Abdomen, 1909, p. 100 
have been relieved by food and the gall-bladder would have been dilated. At operation I found many adhesions around the gall-bladder which contained calculi and a calculus of the size of a cherry stone in the common bile-duct, which I removed after mobilising the duodenum. I closed the opening in the duct and drained the gall-bladder. The patient left the hospital a month later and has remained well ever since.

The special points in the dyspepsia due to biliary calculi are these: its association with flatulent distension; the frequency with which the patient is awakened at night; the pain is not relieved by taking food, and if associated with vomiting this may not relieve it, but vomiting is unusual.

Abdominal examination in these cases may settle the diagnosis; for example, in two of the cases upon which $I$ operated before the onset of colic a distended gall-bladder was distinctly palpable. Tenderness under the right lower ribs is usually present and Murphy's sign may be elicited. Occasionally no physical signs exist.

There remains yet one other group of cases (Group 2) in which it may be impossible to make the diagnosis from a chronic duodenal ulcer. This is not as widely known as it should be. In a paper on Duodenal Ulcer ${ }^{28} \mathrm{I}$ divided the cases according to their symptoms into four groups : (1) latent; (2) those in which a definite diagnosis may be made; (3) those in which the diagnosis of peptic ulcer of stomaoh or duodenum may be made but the symptoms are not clear as to which exists; and (4) those in which the diagnosis is not possible until operation. It is in this last group that difficulties arise. need hardly remind you that rarely duodenal ulcer may be complicated by jaundice and that duodenal ulcer and gallstones may exist together.

A large proportion of all cases of duodenal ulcer give symptoms enabling a certain diagnosis to be made, the most important being hunger pain relieved by taking food and waking the patient in the early hours of the morning. In these cases the ulcer is in the first part of the duodenum. These symptoms are never produced by gall-stones. There are cases, however, in which the symptoms of gall.stones are mimicked closely by those due to an ulcer in the second part of the duodenum. Cases of this nature are, however, unusual.

Among the 46 cases of chronic duodenal ulcer that I have operated on before perforation had taken place the symptoms in three simulated those due to gall-stones and in these the ulcers were situated in the second portion of the duodenum. I have quoted one case in the paper just referred to ; the following is another typical example.

A male, aged 55 years, was sent to melast September with the following history. Since the age of 21 he had been subject to attacks of severe pain in the right side, usually at night, not accompanied by romiting, and not occurring sufficiently often to make him seek medical relief. After the first attack he had no others for ten years. Two or three months before I saw him he had had several severe attacks on following nights accompanied by vomiting. The pain doubled him up and made him sweat and retch. He never tried the effect of taking food because of the retching. In addition he had had for years attacks of discomfort from 2 to 3 hours after food relieved by belching. There was no night pain except with the severe attacks and never hunger pain. The patient was a strong healthy man, and the only physical sign was slight deep tenderness in the right hypochondrium. At the operation I found the gall-bladder and bile-ducts healthy and a large chronic ulcer at the junction of the first and second parts of the duodenum on the anterior wall. After inverting it, I did posterior gastro-jejunostomy.

The particular points in diagnosis that I wished to bring forward may be briefly stated as follows.

1. Attacks of "colic" resembling those due to gall-stones may be the result of moveable kidney or of duodenal nlcer. It may be impossible to make a certain diagnosis without operation.

2. In the majority of patients with gall-stones "indigestion" is present for several years before the first attack of colic. This indigestion has certain featares which often enable a diagnosis to be made but is sometimes suggestive of gastric or duodenal ulcer. An attempt should be made to recognise the condition early, so that operation may be performed at the ideal time while the stones are still in the gall-bladder.

Devonshire-place. W.

28 Medical Press and Circular, April 7th, 1909

\section{CONGENIT:AL ABNORMALITY OF THE} HEART: A CASE OF COR TRILOCULARE BIATRIATUM.

\section{BY JANE I. ROBERTSON, M.B. GlasG.}

THE main anatomical peculiarities in this case of deformity of the heart are as follows: (1) dilated left auricle (2) dilated sinus venosus; persistence of venous valves with marked hypertrophy of the right valve; (3) small right auricle ; obliteration of tricuspid opening; presence of an inter-auricular foramen ; (4) almost complete obliteration of right ventricle; (5) persistence of the bulbus arteriosus; presence of two interventricular foramina; (6) transposition of the large vessels, aorta in front arising from the bulbus arteriosus; pulmonary artery behind arising from the left ventricle ; and (7) persistence of a patent ductus Botalli.

The heart is that of a nine weeks' infant born at full time but always weakly and cyanosed. For the age of the infant the speoimen is of very large size ; it is a broad, short heart, with a rounded apex which is formed entirely by the left ventricle ; there is just the faintest indication of the outline of a small right ventricle high up on the right border. The persistent bulbus arteriosus, from which the transposed aorta arises, forms a marked prominence on the right anterior surface of the heart; to the left of the bulbus branches of the left (or anterior) coronary artery pass towards the right margin, apex, and left margin of the heart. The right auricular appendix is prominent, lying in front of the pulmonary vessel and overlapping the outer side of the bulbus; its tip is curiously bifurcated. Posteriorly the FIG. 1.

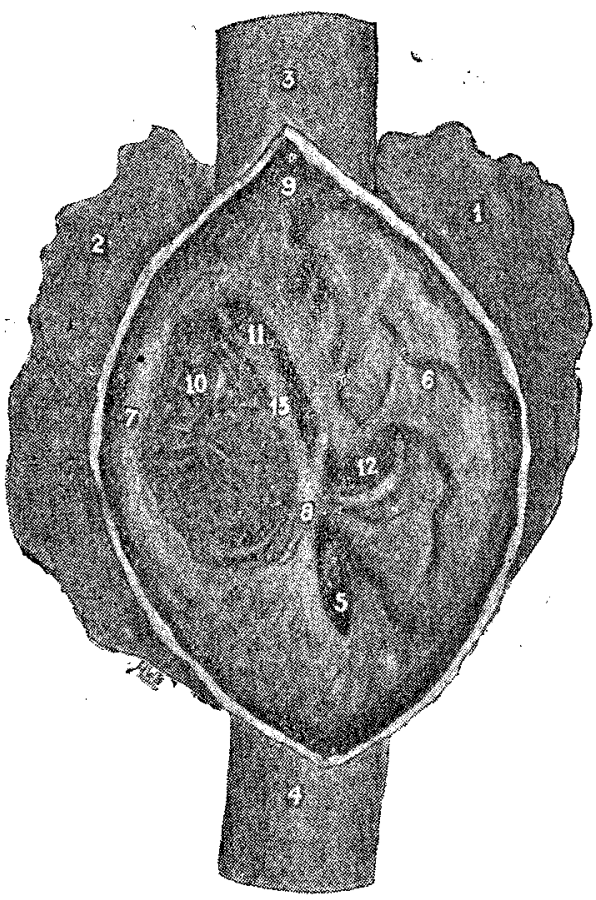

Right auricle. In this illustration the interior of the right auricle is exposed from above and behind by an incision into the sinus venosus extending from the superior to the inferior vena cava. 1. Right auricle. 2. Left auricle 3. Superior vena cava. 4. Inferior vena cava. 5. Coronary sinus. 6. Expanded valvular fold (right venous valve). 7. Flattened band of muscle circling fossa ovalis (left venous valve). 8. Point of junction of posterior ends of the venous valves. 9. Crista terminalis; junction of anterior ends of the venous valves. 10. Fossa ovalis. 11. Inter-auricular foramen. 12. Aperture of communication between sinus venosus and right auricle. 13. Limbus Vieussenii.

most striking features of this specimen are the great degree of dilatation of the left auricle and the wide space between the points of insertion of the right and left pulmonary veins. On the posterior wall of the left ventricle the branches of the right (or posterior) coronary artery run obliquely from right to left. The aorta arises from the persistent balbus arteriosus in front and to the right of the pulmonary artery it arches upwards and to the left, gives off the innominate, left common carotid, thyroidea ima and left subclarian arteries. It then communicates with the left branch of the pulmonary artery by means of a fairly large ductus Botalli and-immediately increasing considerably in calibre-finally 